\title{
Overexpression of Adenosine Kinase in Patients with Epilepsy Associated With Sturge-Weber Syndrome
}

Guoming Luan ${ }^{1 *}$, Xiongfei Wang ${ }^{1,2,}$, Fan Chen ${ }^{2,3}$, Qing Gao ${ }^{2}$, Jian zhou' ${ }^{1}$ Yuguang Guan' ${ }^{1}$ Jing Wang ${ }^{3}$, Feng Zhai', Yin Chen ${ }^{2}$, Tianfu Li ${ }^{2,3,+}$

\begin{abstract}
Background: The Sturge-Weber syndrome (SWS), a vascular disorder with leptomeningeal capillary-venous malformations, is thought to be caused by somatic mutations in the GNAQ gene. However, the relationship between SWS and epileptogenesis is still unknown. Overexpression of adenosine kinase (ADK) has been regarded as a pathologic hallmark of epilepsy. We hypothesize that abnormal expression of ADK, may play an important role in epileptogenesis of SWS patients with refractory epilepsy.
\end{abstract}

Methods: Immunnohistochemistry was used to examine the expression of adenosine kinase (ADK) in cortical specimens from patients with SWS $(n=12)$, and compared with control cortical tissue.

Results: Consistent with previous studies, astrogliosis and the loss of neuronal cells were demonstrated in the lesions area of SWS. In addition, it was first time to reveal the overexpression of ADK, both neuronal and astroglial, with a predominant cytoplasmic localization, in patients with SWS.

Conclusion: These results suggest that over-expression of ADK is a common pathologic hallmark of SWS, which may be crucial in the epileptogenesis of SWS.

\section{Keywords}

Adenosine kinase, Sturge-Weber syndrome, Epilepsy

\section{Introduction}

The Sturge-Weber syndrome (SWS), also known as 'the kiss of angel', is a congenital neurocutaneous disorder caused by somatic mosaic mutations in the GNAQ gene, disrupting capillary venous vessels in the leptomeninges of the brain and choroid [1]. The patient with SWS often demonstrates a port-wine stain ipsilateral to the vascular malformation.
The clinical manifestation of SWS is focal neurological deficits, epileptic seizures, or mental retardation that can be regarded as comorbidity of epilepsy [2,3]. It is generally accepted that SWS, with affected skin in distribution of the ophthalmic branch of the trigeminal nerve, is always associated with catastrophic childhood epilepsy [4]. The neuropathological hallmarks of SWS consist of predominantly leptomeningeal

'Department of Neurosurgery, Center of Epilepsy, Beijing Institute for Brain Disorders, Beijing Key Laboratory of Epilepsy Research, Sanbo Brain Hospital, Capital Medical University, Beijing, China

${ }^{2}$ Department of Brain Institute, Center of Epilepsy, Beijing Institute for Brain Disorders, Beijing Key Laboratory of Epilepsy Research, Sanbo Brain Hospital, Capital Medical University, Beijing, China

${ }^{3}$ Department of Neurology, Center of Epilepsy, Beijing Institute for Brain Disorders, Sanbo Brain Hospital, Capital Medical University, Beijing, China

${ }^{+}$Author for correspondence: Tianfu Li, MD., Ph D, Professor and Chief Physician in Neurology, Vice director of Brain Institute \& Epilepsy Center, Sanbo Brain Hospital, Capital Medical University. Xiangshan Yikesong 50, Haidian district, Beijing, 100093, Tel: +86 1062856761; Fax: +86-10-62856902; E-mail: tianfuli66@126.com 
angiomatosis, cortical atrophy with clodded calcareous deposits in the cortex and white matter, neuronal loss and astrogliosis [5,6].

In terms of epileptogenic mechanism, it still remains unclear, electrophysiologically or histopathologically. Chronic ischemia in the cortical areas affected by leptomeningeal angiomatosis has been usually considered to be the epileptogenic mechanism [7]. Increasing clinical evidence from specimen surgically resected from patients with drug-resistant epilepsy, including temporal lobe epilepsy $[8,9]$, Rasmussen encephalitis [10], astroglial tumorrelated epilepsy [11], and focal cortical dysplasia [12], demonstrated that overexpression of astroglial adenosine kinase (ADK) contributing to seizures generation in human chronic epilepsy. However, in our study, it is first time to literarily report a link between adenosine and SWS.

Epileptic seizures and cognitive impairment are prominent clinical features of SWS and recent data suggest that the dysregulation of adenosinergic mechanism is involved in the development of epilepsy and its comorbidities $[13,14]$. Adenosine is an endogenous purine nucleoside that modulates a wide range of physiologic functions. Most notable among its many roles is its importance in inhibiting seizures [13,15-23], and restoring cognitive function when cognition is impaired secondary to epilepsy [13,24].

Based on the evidence that adenosine signaling plays a crucial role in preventing seizures and inflammation, we hypothesized that the epileptogenic mechanisms underlying SWS might be related to changes in $\mathrm{ADK}$ expression and that those changes might be associated with the development of epilepsy in patients with SWS.

\section{Material and Methods}

\section{- Patients and diagnosis}

The local Ethics Committee (Beijing Sanbo Hospital, Capital Medical University, China) approved all studies and clinical investigations were conducted according to the declaration of Helsinki. Informed consent was obtained from all participants or their parents or legal guardians.

In this study, surgical brain tissue samples were obtained from banked on cases previously diagnosed with SWS, including from their resection specimens neuropathological analyses. Presurgical evaluation in Beijing Sanbo Hospital included MRI (spin-echo T1-weighted axial and T2-weighted axial, coronal sequences and fluid-attenuated inversion recovery images with 5-mm-thick axial, sagittal, coronal sections), Scalp Video-electroencephalography (vEEG), intracranial EEG monitoring, seizures semiology analysis as well as neuropsychological test. Interictal/ictal scalp electroencephalography (EEG) was recorded using a video-EEG monitoring system (Nicolet vEEG; Viasys Healthcare, Madison, USA), with electrodes placed according to the international 10-20 system for all patients. The duration of videoEEG monitoring ranged from 1-3 days, and at least three habitual seizures were recorded. The main ictal manifestations were categorized according to the new semiological seizures classification $[25,26]$.

Twelve patients with SWS were identified in the neuropathology archives at Sanbo Brain Hospital, diagnosed between January 2006 and August 2014. There were 5 males and 7 females, with 7 right lateralization and 5 left lateralization, with a mean age of seizures onset of $3.65 \pm 5.20$ years old (range $0.25-14$ ), a mean age at surgery of $16.11 \pm 13.46$ years old (range 1.00-49.00), and a mean interval between the seizures and first surgery $12.46 \pm 13.65$ years (range 0.75 47.00). The first clinical manifestation of all 12 patients was seizures. All patients were observed neither family history nor perinatal history. Clinical information of 12 patients with SWS was summarized in Table 1. All brain tissues were obtained from 12 SWS patients during operation and then paraffin-embedded. In addition, we studied control brain samples obtained at autopsy from 6 patients without a history of seizures or other neurologic diseases. All autopsies were performed within 12 hours after death.

\section{- Tissue preparation}

Formalin-fixed, paraffin-embedded tissue samples were sectioned at $4 \mu \mathrm{m}$ and mounted on precoated glass slides (Star Frost, Waldemar Knittel $\mathrm{GmbH}$, Braunschweig, Germany). Sections of all specimens were processed for hematoxylin eosin (HE), as well as for immunohistochemical stainings for glial marker glial fibrillary acidic protein (GFAP), the neuronal marker neuronal nuclear protein $(\mathrm{NeuN})$, and $\mathrm{ADK}$, which are described below.

\section{- Immunohistochemistry}

Antibodies to glial fibrillary acidic protein (monoclonal mouse, DAKO; Glostrup, 


\begin{tabular}{|c|c|c|c|c|c|c|}
\hline $\begin{array}{l}\text { Patient } \\
\text { number }\end{array}$ & Gender & Lateralization & $\begin{array}{l}\text { Age at first } \\
\text { seizure }\end{array}$ & Seizure types & $\begin{array}{l}\text { Interval between } \\
\text { the seizure and first } \\
\text { surgery }\end{array}$ & Age at surgery \\
\hline 1 & Female & $\mathrm{R}$ hemisphere & 4 months & $\begin{array}{l}\text { CPS } \\
\text { GTCS }\end{array}$ & 44 months & 4 years \\
\hline 2 & Male & L temporal & 6 months & CPS & 27.5 years & 28 years \\
\hline 3 & Male & $\mathrm{R}$ hemisphere & 3 months & GTCS & 9 months & 1 years \\
\hline 4 & Male & L parietal+ occipital & 3 years & SPS & 24 years & 27 years \\
\hline 5 & Female & L temporal+ parietal+occipital & 3 years & CPS & 10 years & 13 years \\
\hline 6 & Female & R temporal+ occipital & 5 months & CPS & 5.58 years & 6 years \\
\hline 7 & Male & R occipital & 1 years & $\begin{array}{l}\text { SPS } \\
\text { GTCS }\end{array}$ & 6 years & 7 years \\
\hline 8 & Female & L occipital & 16 years & SPS GTCS & 3 years & 19 years \\
\hline 9 & Male & R temporal+ parietal+occipital & 3 years & GTCS & 19 years & 22 years \\
\hline 10 & Female & R temporal+ parietal+occipital & 14 years & GTCS & 2 years & 16 years \\
\hline 11 & Female & L hemisphere & 4 months & $\begin{array}{l}\text { CPS } \\
\text { GTCS }\end{array}$ & 1 years & 16 months \\
\hline 12 & Female & R parietal+occipital & 2 years & SPS & 47 years & 49 years \\
\hline
\end{tabular}

Denmark, 1:4000) neuronal nuclear protein (NeuN; mouse clone MAB377, IgG1; Chemicon, Temecula, CA; 1:2000), and ADK (Provided by professor Detlev Boison, R.S. Dow Neurobiology Laboratories, Legacy Research Institute, Portland, Oregon, USA. polyclonal rabbit, 1:500) were used in the routine immunohistochemical analysis of SWS human specimens. Double-label immunocytochemistry was performed as described previously $[12,18]$. Sections after incubation with primary antibodies, were incubated for $2 \mathrm{~h}$ at room temperature with Alexa Fluor 568 and Alexa Fluor 488 (antirabbit IgG or antimouse IgG; 1:200; Molecular Probes, Eugene, OR). Images were visualized using a Leica microscope under Ex/Em wavelength of 500/550nm (green), collected using an Optronics DEI-750 three-chip camera equipped with a BQ 8000 sVGA frame grabber, and analyzed using Bioquant software (Nashville, TN).

A semiquantitative immunohistochemistry gradient analysis of the test tissue from area was constructed for analysis of differences in ADK immunostaining. For the immunohistochemical detection of ADK immunostaining, we followed our previously published procedures with modification. Levels of $\mathrm{ADK}$ in each analysis field $(434 \mu \mathrm{m}$ X $326 \mu \mathrm{m})$ were initially measured as arbitrary density units, and subsequently expressed as a percent change from control ADK. Corresponding fields from each SWS patient $(n=12)$ and controls $(n=6)$ were analyzed by scanning $\mathrm{ADK}$ immunofluorescence on $\mathrm{DAB}$ stained slices using a Kodak imaging device.

\section{Statistical analysis}

Quantitative data were analyzed using GraphPAD Prism software (GraphPAD Software, La Jolla, CA). Data for ADK immunohistochemistry quantification analyzed using two-tailed Student t-test. $\mathrm{p}<0.05$ was considered significant.

\section{Results}

\section{- Clinical manifestations and pathologic features}

The clinical manifestations of the cases included in this study are summarized in Table 1 . The seizures types of patients in the present study included simple partial seizures (SPS), complex partial seizures (CPS) and secondary generalized tonic clonic seizures (GTCS). All 12 patients underwent surgical resection for treatment of refractory epilepsy, including lobe resectomies, multi-lobe resectomies and functional hemispherectomies. None patient presented lesions covering bilateral hemisphere, which was regarded as surgical contraindication [27]. Figure 1(A-G) demonstrated the example of procedures for surgical resection in patient with SWS. MRI was abnormal in all patients. Gadolinium-enhanced image typically shows leptomeningeal enhancement before surgery treatment in all cases (Figure 1B-D). T2weighted fluid-attenuated inversion recovery image reveals changes after resection surgery (Figure 1H-J). Leptomeningeal enhancement and calcification were found in every patient by MRI and CT scan. The $67.7 \%$ (8/12) patients presented occipital involvement, and 7 of them 

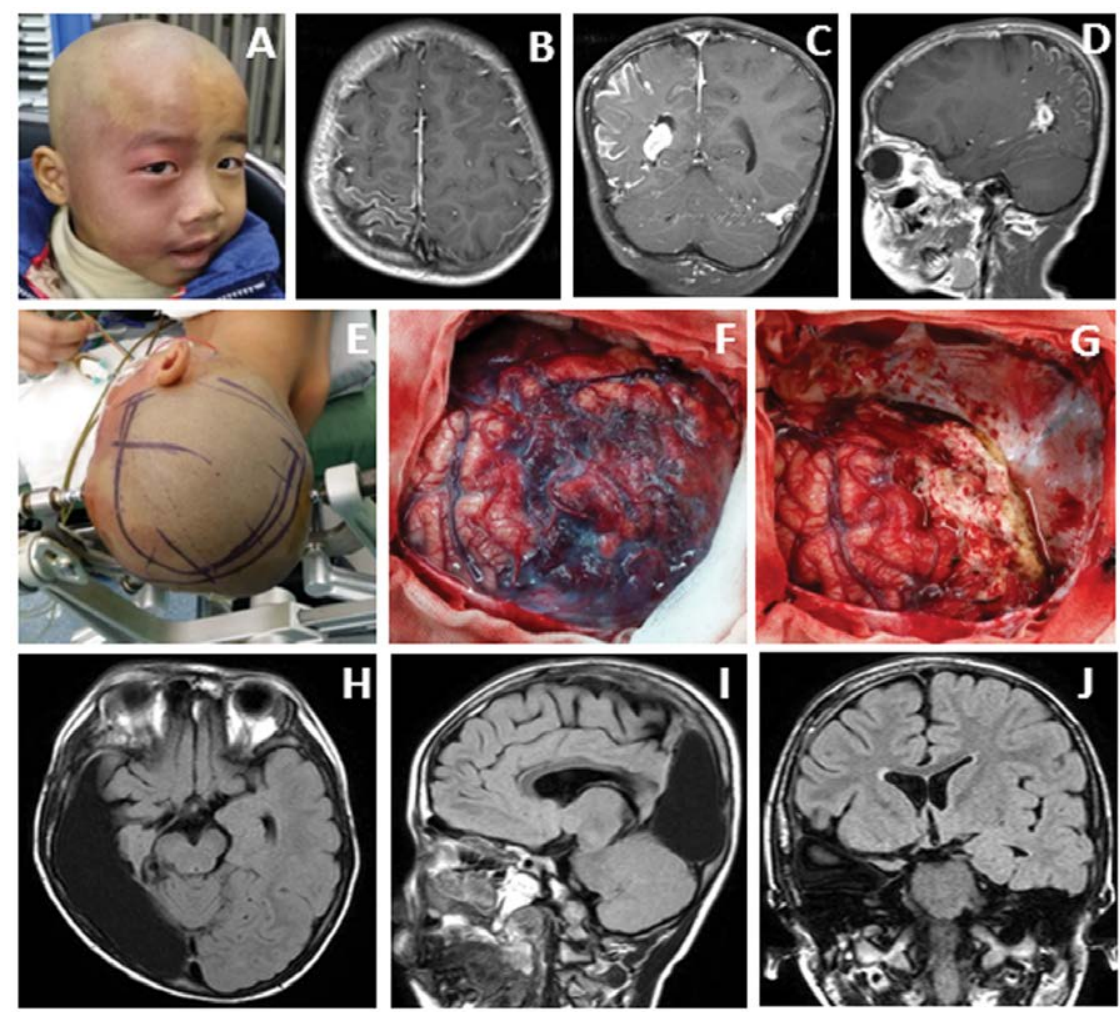

Figure 1: Example of surgical treatment for patient with SWS.

(A) Port wine stain in distribution of trigeminal nerve, mainly ophthalmic branch presented in the patient 7. (B-D) Presurgical evaluation of gadoliniumenhanced T1-weighted image of MR showed enhancing leptomeningeal vessels in right occipital lobe. (E) Frontotemporoparietal approach in lateral decubitus was applied in the operation. (F) Bipolar electro-coagulation on functional cortex in insular. (G) Resection of an epileptogenic region in right frontotemporoparietal area. $(\mathrm{H}-\mathrm{J})$ The postoperative image of T2-weighted fluid-attenuated inversion recovery MR in 6 months confirmed the range of resectomy.

demonstrated visual field cut. By visual analyzing the image of MRI and CT scan, we found that $25 \%(3 / 12)$ patients, with abnormal capillary venous vessels affecting hemisphere of cerebrum, presented with hemiplegia.

Microscopically, Neuronal loss (Figure 2A, B, arrowheads) and clodded calcareous deposists (Figure 2A, B, arrows) were demonstrated in the grey matter. Leptomeningeal angiomatosis characterized by excessive subarachnoidal vessels was found in every SWS patient (Figure 2C, D), which is consistent with previous study [28]. Large dysmorphic neurons (Figure 2E, F, arrows), with obvious different morphology comparing with normal pyramidal cells (Figure $2 \mathrm{E}, \mathrm{F}$, arrowheads), were detected in the grey matter. Reactive astrogliosis was demonstrated in the grey matter (Figure 2G, arrowheads) and white matter (Figure 2H, arrows).

\section{- Upregulation of ADK in SWS}

Consistent with previous studies [10,12,24], in white matter of the control group, $\mathrm{ADK}$ immunoreactivity was present in sparse astroglial cells with only a weak staining (Figure 3F); and no positive staining in neuronal cells (Figure 3E). In SWS specimens, ADK immunoreactivity was remarkably observed in the reactive astroglial cells in the white matter within the lesion area, with a predominant cytoplasmic localization (Figure 3C, D, inset, arrows). In addition, ADK was also observed in a subpopulation of neuronal cells in the lesion area, with a predominant cytoplasmic localization (Figure 3A, B, inset, arrows). The density of ADK immunoreactivity of white matter $(n=12)$ and grey matter $(n=12)$ within the lesion area indicated a significant increase $(\mathrm{p}<0.05$, respectively) compared with controls $(n=6)$ (Figure 4). Double labeling of ADK with GFAP confirmed that ADK expressed in GFAP-positive reactive astrocytes (Figure 5A$\mathrm{D}$, arrows).

\section{Discussion}

The clinical manifestation of SWS is focal neurological deficits, epileptic seizures, or mental 

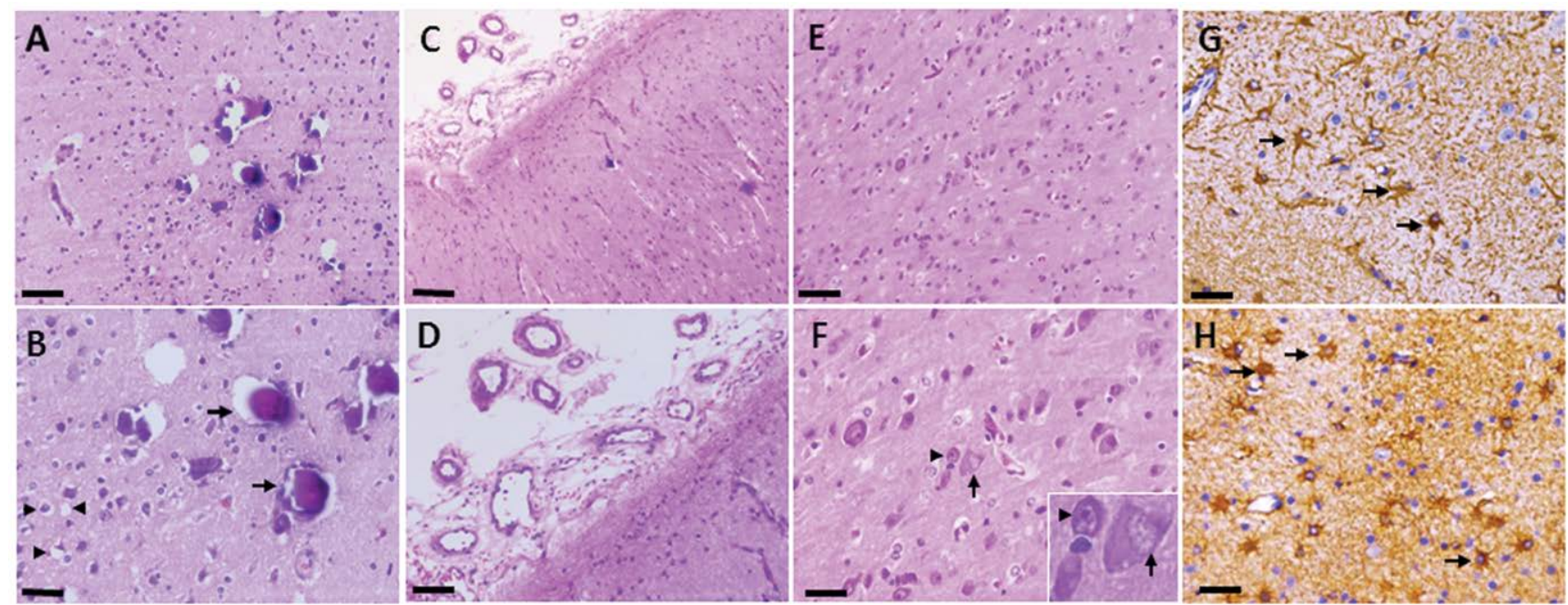

Figure 2: Neuropathological features in SWS.

(A-B) Neuronal loss (B, arrowheads) and clodded calcareous deposists (B, arrows) were demonstrated in the grey matter. (C-D) Leptomeningeal angiomatosis was detected with the feature of excessive subarachnoidal vessels. (E-F) Large dysmorphic neurons (F, inset, arrows) were demonstrated in the grey matter with obvious different morphology comparing with normal pyramidal cells (F, inset, arrowheads). (G-H) Reactive astrogliosis was exhibited in the grey matter (arrowheads) and white matter (arrows). Scale bars: $(A, E, G, D)=50 \mu \mathrm{m} . C=100 \mu \mathrm{m} .(B, F, H)=25 \mu \mathrm{m}$.

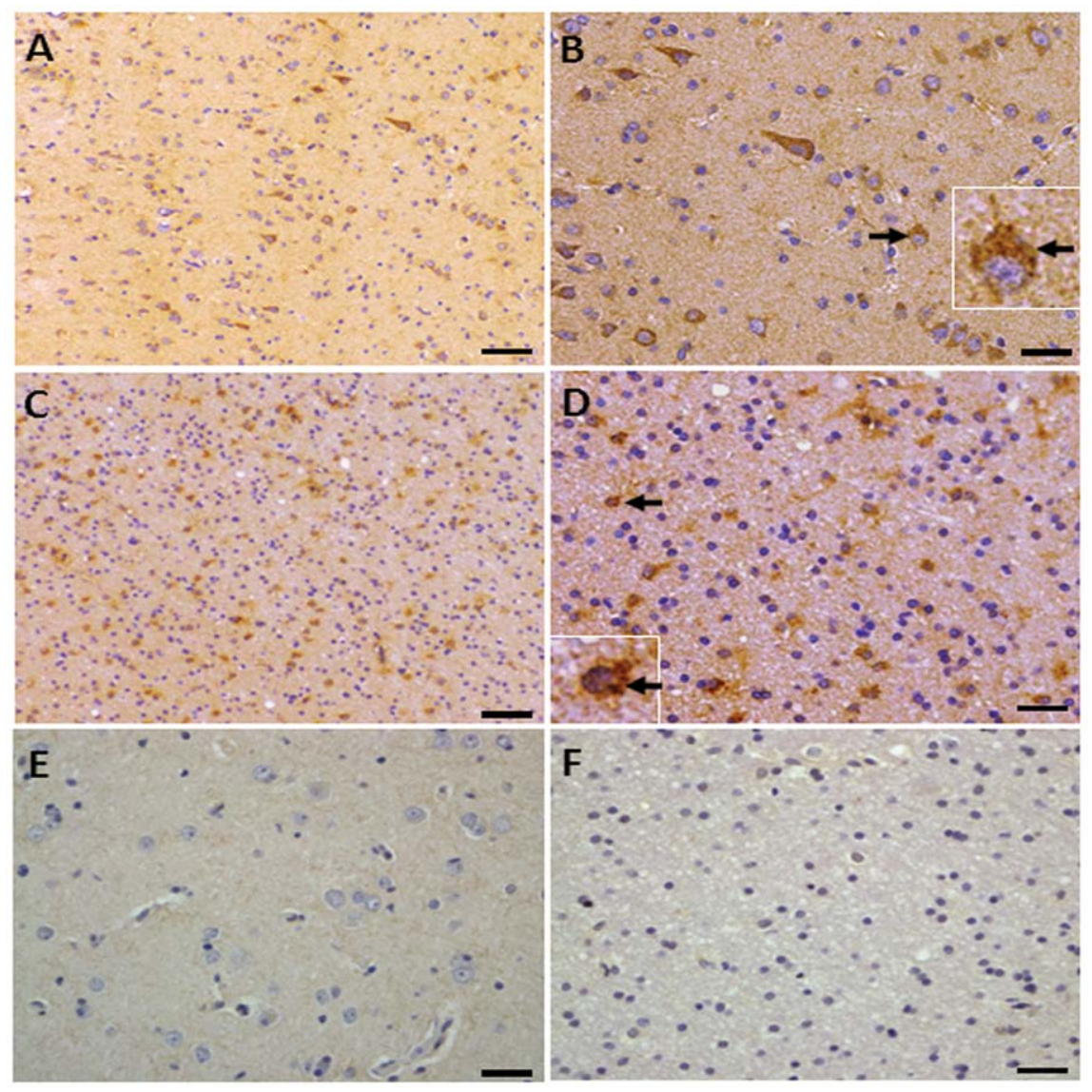

Figure 3: Upregulation of ADK in lesion areas in patients with SWS.

(A-B) There is marked ADK immunoreactivity in a subpopulation of the remnant of neurons (B, inset, arrows) within the lesion area in patients with SWS. (C-D) There is marked $A D K$ immunoreactivity in reactive astrocytes ( $D$, inset, arrows) within the lesion area in patients with SWS. (E-F) There is weak immunostaining for ADK in control cortical gray matter (E) and sparse glial cells in control white matter (F). Scale bars: $(A, C)=50 \mu m$. (B, D, E, F) $=25 \mu m$. 


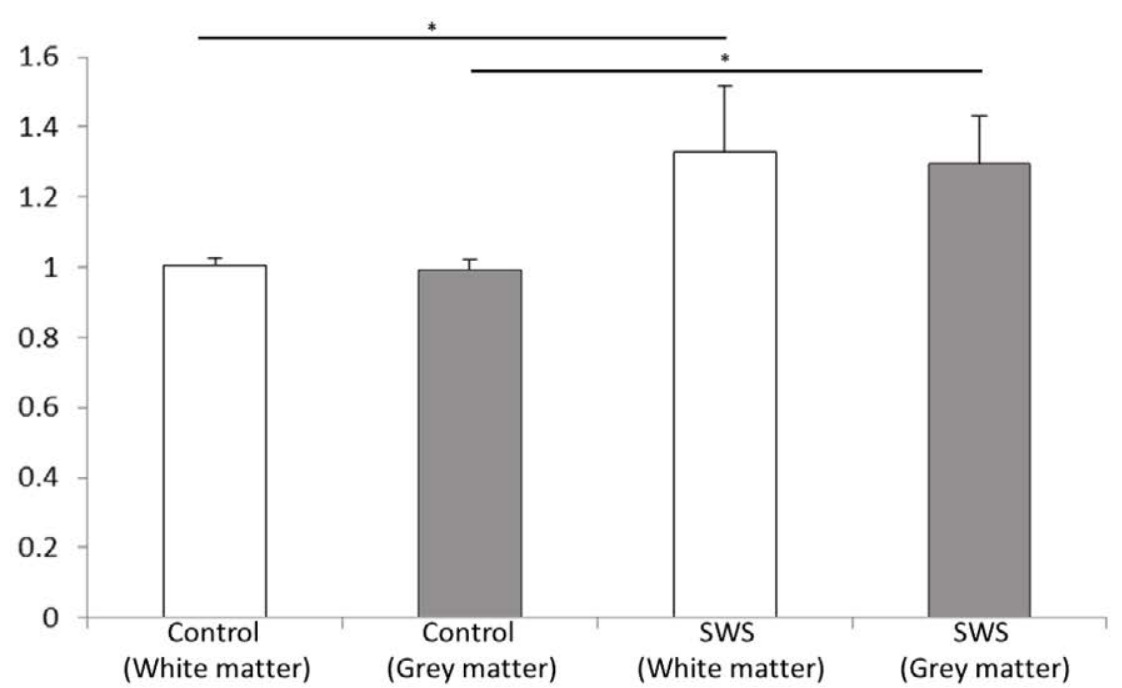

Figure 4: Density of ADK immunoreactivity in patients with SWS.

The density of ADK immunoreactivity of white matter $(n=12)$ and grey matter $(n=12)$ within the lesion area indicated a significant increase compared with controls $(n=6) .\left({ }^{*} p<0.05\right)$.
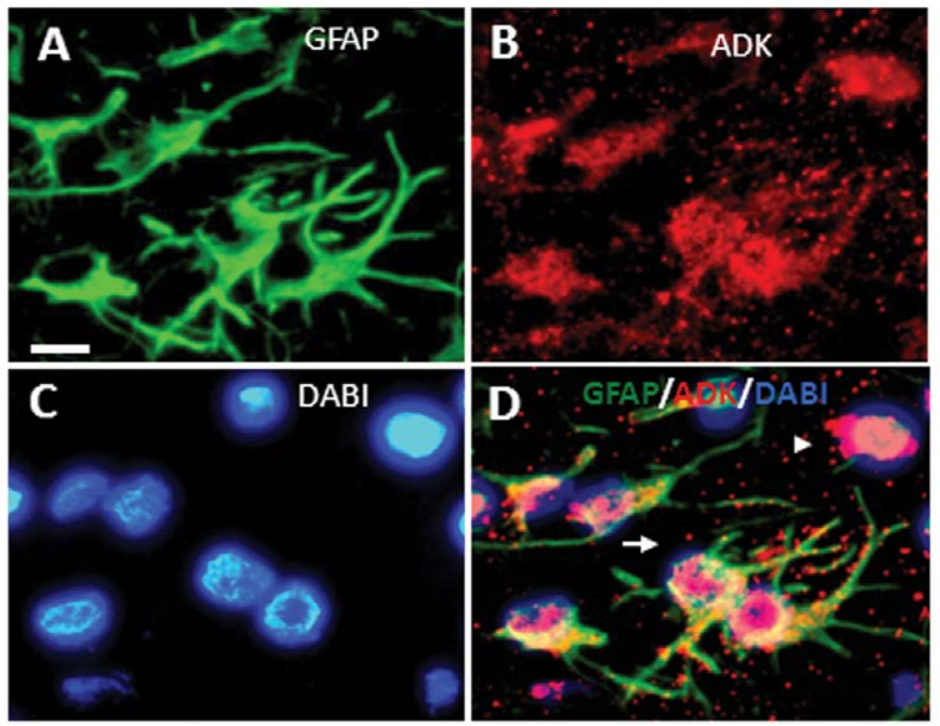

Figure 5: Double labeling for ADK with GFAP in lesion areas in patients with SWS.

Co-localization of ADK (B, red) with GFAP ( $A$, green) and DABI (C, green) confirms expression of ADK, with a predominant cytoplasmic expression, in astroglial cells ( $D$, arrows). The cell with negative GFAP, positive DAPI and positive ADK ( $D$, arrowheads) could be the ADK positive neuron. Scale bars: $15 \mu \mathrm{m}$.

retardation that can be regarded as comorbidity of epilepsy [2,3]. The onset of epilepsy in infancy in patients with SWS is often catastrophic, with medically intractable seizures, hemiparesis, and severe cognitive impairment [29]. A series of studies have elucidated that adenosine dysfunction is associated with epileptogenesis, especially disruption of adenosine homeostasis caused by the alterations of major adenosine clear enzyme ADK. Because ADK activity is the principal factor controlling adenosine metabolism in the brain, minor changes in ADK activity translate rapidly into major changes in adenosine [30]. Dysregulation of ADK plays a significant role in the processes that turn a normal brain into an epileptic brain [14]. Experimental researches demonstrate that overexpression of ADK per se might be sufficient to trigger electrographic seizures $[8,15,18,19,22]$. Clinical evidence also demonstrates that $\mathrm{ADK}$ as a common pathologic hallmark and a target for treatment in intractable epilepsy such as temporal epilepsy $[8,9]$, tumor- 
related epilepsy [11], Rasmussen encephalitis $[10,24]$, and focal cortical dysplasia [12].

In the present study, reactive astrgliosis, the pathological characteristic features of SWS and astroglial overexpression of ADK was demonstrated in all patients. Astroglial overexpression of ADK, with a predominant cytoplasmic localization, has been demonstrated to be associated with reactive astrogliosis and consequential development of adenosine deficiency, which has been proved to be a hallmark in epileptogenesis by experiments and clinical evidence $[8,10,12,13,18,24]$. Considering the relationship between astroglial ADK overexpression and gliosis, we hypothesize that ADK may be a potential target for SWS patient with refractory epilepsy.

In our study, expression of ADK was evidenced in a subpopulation of neuronal cells in the lesion area in patients with SWS. Most of the neuronal cells in the human brain are the terminally differentiated cells and lack the ADK expression. Under physiological condition, neuronal cells constitute a major source for direct release of adenosine. However, functional implication of neuronal ADK expression has not been explored. Analysis on human specimens from autopsy control and surgical control demonstrated ADK immunoreactivity was present in sparse glial cells with only a weak staining, and lack of expression in most of the neurons in the cortex [12,24,31]. Our recent study demonstrated no difference in ADK expression between children (more than 3 years) and adult autopsy control cortex. Further study is needed on younger children (eg 1 month, 6 months, 1 year, 2 years, and 3 years) for the developmental and age-related differences [12]. The hypothesis on neuronal expression of ADK is that alteration during early stage of developmental microenvironment may give rise to a revertant fetal expression, which means impairment of temporal transient expression pattern of neuronal ADK from fetal to postnatal brains [24].

In addition, $\mathrm{ADK}$ also plays an important role in modulation of cognitive process. This is evidenced by transgenic overexpression of $\mathrm{ADK}$ in the brain of mice (Adk-tg mice) caused prominent cognitive impairment on several levels $[31,32]$. In clinical practice, cognitive deficits, or mental retardation, may be considered as one of most common comorbidities of refractory epilepsy, especially for children [3,33]. Our recent study has demonstrated that vagus nerve stimulation
(VNS) or deep brain stimulation (DBS) might improve neuropsychological outcomes, including language, memory, perception $[34,35]$. Consequently, we hypothesize that VNS or DBS may be an alternative treatment for SWS patients with refractory epilepsy, especially for those who cannot receive surgical resection.

We acknowledge limitations to our study. It is a relatively small cohort of patients, which makes impossible to avoid the bias brought by clinical variables. In terms of adenosine system, adenosine receptors have not been involved in the current study. Therefore, further investigation for the expression, regulation, function of adenosine receptors should be conducted.

In conclusion, overexpression of $\mathrm{ADK}$, both neuronal and astroglial, plays an important role in the epileptogenesis of SWS. Furthermore, the therapeutic strategy of adenosine augment should be considered and explored deeply, which may greatly improve the prognosis of SWS patients with contraindication for surgical resection.

\section{Abbreviations \\ SWS: Sturge-Weber syndrome; ADK: adenosine kinase; SPS: simple partial seizure; CPS: complex partial seizure; GTCS: generalized tonic clonic seizure; EEG: electroencephalography; GFAP: glial fibrillary acidic protein; VNS: vagus nerve stimulation; DBS: deep brain stimulation;}

\section{Acknowledgment}

The authors thank Professor Detlev Boison (RS Dow Neurobiology Laboratories, Legacy Research Institute, Portland, OR) for his kind and generous gift of ADK antibody. This Project was supported by the Grant from the BIBDPXM2013_014226_07_000084, National Natural Science Foundation of China (81571275), Scientific Research Common Program of Beijing Commission of Education (KM201410025027). We confirm that we have read the Journal's position on issues involved in ethical publication and affirm that this report is consistent with those guidelines.

\section{Authors' Contributions}

Immunohistochemistry and the analysis of the data were performed by Wang X, Luan G, Chen $\mathrm{F}$ and Chen Y. Wang $\mathrm{X}$ and Chen F helped Li $\mathrm{T}$ in drafting and preparing the manuscript for submission. The overall experimental design was 
conceived and supervised by Li T. Zhou J, Guan Y, Feng Z, Wang J helped in the selection and collection of brain tissues. All authors read and approved the final manuscript.

\section{Competing Interests}

The authors declare that they have no competing interests.

\section{References}

1. Shirley MD, Tang $\mathrm{H}$, Gallione $\mathrm{CJ}$, et al. Sturge-Weber syndrome and port-wine stains caused by somatic mutation in GNAQ. N. Engl. J. Med 368(21), 1971-1979 (2013).

2. Comi A. Current Therapeutic Options in Sturge-Weber Syndrome. Semin. Pediatr. Neurol 22(4), 295-301 (2015).

3. Li T. Epilepsy and Associated Comorbidities. Neuropsychiatry (London) e1(01), 1-3 (2017).

4. Otsuki T, Kim HD, Luan G, et al. Surgical versus medical treatment for children with epileptic encephalopathy in infancy and early childhood: Results of an international multicenter cohort study in Far-East Asia (the FACE study). Brain. Dev 38(5), 449-460 (2016).

5. Pinto $A L$, Chen $L$, Friedman $R$, et al. Sturge-Weber Syndrome: Brain Magnetic Resonance Imaging and Neuropathology Findings. Pediatr. Neurol 58(1), 5-30 (2016).

6. Norman MG, Schoene WC. The ultrastructure of Sturge-Weber disease. Acta. Neuropathol. Mar 37(3), 199-205 (1977).

7. Thomas-Sohl KA, Vaslow DF, Maria BL. Sturge-Weber syndrome: a review. Pediatr. Neurol 30(5), 303-310 (2004).

8. Masino SA, Li T, Theofilas P, et al. A ketogenic diet suppresses seizures in mice through adenosine $\mathrm{A}(1)$ receptors. J. Clin. Invest 121(7), 2679-2683 (2011).

9. Aronica $E$, Zurolo $E$, lyer $A$, et al. Upregulation of adenosine kinase in astrocytes in experimental and human temporal lobe epilepsy. Epilepsia 52(9), 1645-1655 (2011).

10. Luan G, Gao Q, Guan Y, et al. Upregulation of adenosine kinase in Rasmussen encephalitis. J. Neuropathol. Exp. Neurol 72(11), 1000-1008 (2013).

11. de Groot M, lyer A, Zurolo E, et al. Overexpression of ADK in human astrocytic tumors and peritumoral tissue is related to tumor-associated epilepsy. Epilepsia 53(1), 58-66 (2012).

12. Luan G, Gao Q, Zhai F, et al. Adenosine kinase expression in cortical dysplasia with balloon cells: analysis of developmental lineage of cell types. J. Neuropathol. Exp. Neurol 74(2), 132-147 (2015).
13. Li T, Gao Q, Luan G. Adenosine dysfunction in Rasmussens encephalitis. Neuropsychiatry (London) 6(5), 280-285 (2016).

14. Boison D. Adenosinergic signaling in epilepsy. Neuropharmacology 104(1), 131-139 (2016).

15. Li T, Lytle N, Lan JQ, et al. Local disruption of glial adenosine homeostasis in mice associates with focal electrographic seizures: a first step in epileptogenesis? Glia 60(1), 83-95 (2012).

16. Szybala C, Pritchard EM, Lusardi TA, et al. Antiepileptic effects of silk-polymer based adenosine release in kindled rats. Exp. Neurol 219(1), 126-135 (2009).

17. Li T, Ren G, Kaplan DL, et al. Human mesenchymal stem cell grafts engineered to release adenosine reduce chronic seizures in a mouse model of CA3-selective epileptogenesis. Epilepsy. Res 84(2-3), 238-241 (2009).

18. Li T, Ren G, Lusardi T, et al. Adenosine kinase is a target for the prediction and prevention of epileptogenesis in mice. J. Clin. Invest 118(2), 571-582 (2008).

19. Li T, Lan JQ, Boison D. Uncoupling of astrogliosis from epileptogenesis in adenosine kinase (ADK) transgenic mice. Neuron. Glia. Biol 4(2), 91-99 (2008).

20. Ren G, Li T, Lan JQ, et al. Lentiviral RNAi-induced downregulation of adenosine kinase in human mesenchymal stem cell grafts: a novel perspective for seizure control. Exp. Neurol 208(1), 26-37 (2007).

21. Li T, Steinbeck JA, Lusardi T, et al. Suppression of kindling epileptogenesis by adenosine releasing stem cell-derived brain implants. Brain. May 130(Pt 5), 1276-1288 (2007).

22. Li T, Quan Lan J, Fredholm BB, et al. Adenosine dysfunction in astrogliosis: cause for seizure generation? Neuron. Glia. Biol 3(4), 353-366 (2007).

23. Fedele DE, Li T, Lan JQ, et al. Adenosine A1 receptors are crucial in keeping an epileptic focus localized. Exp. Neurol 200(1), 184-190 (2006).

24. Luan G, Wang X, Gao Q, et al. Upregulation of Neuronal Adenosine A1 Receptor in Human Rasmussen Encephalitis. J. Neuro- pathol. Exp. Neurol 76(8), 720-731 (2017).

25. Fisher RS, Cross JH, French JA, et al. Operational classification of seizure types by the International League Against Epilepsy: Position Paper of the ILAE Commission for Classification and Terminology. Epilepsia 58(4), 522-530 (2017).

26. Fisher RS, Cross JH, D'Souza C, et al. Instruction manual for the ILAE 2017 operational classification of seizure types. Epilepsia 58(4), 531-542 (2017).

27. Arzimanoglou AA, Andermann F, Aicardi J, et al. Sturge-Weber syndrome: indications and results of surgery in 20 patients. Neurology 55(10), 1472-1479 (2000).

28. Maton B, Krsek P, Jayakar P, et al. Medically intractable epilepsy in Sturge-Weber syndrome is associated with cortical malformation: implications for surgical therapy. Epilepsia 51(2), 257-267 (2010).

29. Simonati A, Colamaria V, Bricolo A, et al. Microgyria associated with Sturge-Weber angiomatosis. Childs. Nerv. Syst 10(6), 392395 (1994).

30. Boison D. Adenosine kinase, epilepsy and stroke: mechanisms and therapies. Trends. Pharmacol. Sci 27(12), 652-658 (2006).

31. Luan G, Gao Q, Zhai F, et al. Upregulation of HMGB1, toll-like receptor and RAGE in human Rasmussen's encephalitis. Epilepsy. Res 123(1), 36-49 (2016).

32. Yee BK, Singer $P$, Chen JF, et al. Transgenic overexpression of adenosine kinase in brain leads to multiple learning impairments and altered sensitivity to psychomimetic drugs. Eur. J. Neurosci 26(11), 3237-3252 (2007).

33. Wang X, He X, Luan G. Comorbidities of Refractory Epilepsy and the Update Mechanism. Neuropsychiatry (London) S1(007), 45-48 (2017).

34. Guan Y, Chen S, Zhang Y, et al. The Seizure and Cognitive Outcome of Anterior Thalamic Nucleus Deep Brain Stimulation for Patients with Intractable Epilepsy. Neuropsychiatry (London) S1(001), 4-9 (2017).

35. Wang X, Gao Q, Zhou J, et al. The Neuropsychological Efficacy of Vagus Nerve Stimulation in 56 Children with Catastrophic Epilepsy. Neuropsychiatry (London) 7(4), 387-392 (2017). 